\title{
Successful modeling, design, and test of electron cyclotron resonance ion sources
}

\author{
A. Heinen, M. Rüther, J. Ducrée, J. Leuker, J. Mrogenda, H. W. Ortjohann, E. Reckels, \\ Ch. Vitt, and H. J. Andrä \\ Inst. für Kernphysik, University of Münster, Wilh. Klemm Str. 9, D-48149 Münster, Germany
}

(Presented on 10 September 1997)

\begin{abstract}
Plasmas in electron cyclotron resonance ion sources (ECRIS) are collisionless and can therefore be simulated by only following the motion of electrons in the confining static magnetic and oscillating microwave field of ECRIS. The experimental performance of three different ECRIS is successfully compared to calculated spatial electron (plasma) and electron energy density as well as to the energy spectrum and the average energy of the electrons. Further simulations suggest a new and better design of an ECRIS, the good experimental performance of which corresponds to the predictions.

(C) 1998 American Institute of Physics. [S0034-6748(98)68802-X]
\end{abstract}

Electron cyclotron resonance ion sources (ECRIS) have had an important impact on recent experiments in nuclear, atomic, and ion source physics, well summarized in a compendium. ${ }^{1}$ They produce continuous currents of singly to highly charged ions at very good brightness. Their conception without hot filaments allows permanent operation over months. ECRIS have thus a great potential for applications in well established and future ion beam technologies if the ion beam densities can further be improved. To this end we have modeled the ECR plasma and have compared the results to the output ion currents of three ECRIS with different magnetic field configurations and microwave resonance modes. We are able to establish for the first time a direct correlation between model calculations and the performance of an ECRIS.

ECRIS plasmas at densities of $n \leqslant 10^{12} \mathrm{~cm}^{-3}$ are known to be collisionless. ${ }^{1}$ The total rate for all electron-electron, electron atom(ion) collisions is dominated by the ionization of neutrals and is then of the order of $\leqslant 10^{6} \mathrm{~s}^{-1}$ at $100 \mathrm{eV}$ and $\leqslant 10^{5} \mathrm{~s}^{-1}$ at $10 \mathrm{keV}$. A great fraction of the electrons is therefore collisionless for times shorter than $10^{-6} \mathrm{~s}$ and can be ECR heated while the ions stay cold at $\leqslant 2 \mathrm{eV}$ per charge for the ECRIS presented. In such an extreme nonthermal equilibrium situation, the free-electron dynamics determines the spatial behavior of the plasma in its magnetic confinement. The ions actually follow the electron density as a neutralizing background. All collective plasma phenomena can thus be neglected and only the free motion of electrons has to be taken into account in order to obtain the evolution of a plasma inside an ECRIS.

The low cost and compact ECRIS I, II, and III considered here are NeFeB-permanent magnet sources which have an axial mirror field created by two radially magnetized rings of length $\Delta z=60 \mathrm{~mm}$ each (i.d. $=45$, o.d. $=105$ for I, o.d. $=165$ for II and III) with opposite magnetization and a radial confinement created by a hexapole of classical design for I (six poles, $\Delta z=90 \mathrm{~mm}$ ) or of Halbach design for II and III (i.d. $=45$, o.d. $=65, \Delta z=106 \mathrm{~mm}$ ). ${ }^{2}$ The axial mirror field is modified by additional permanent magnet rings in the central region to yield the three types of magnetic field $B_{z}$ on the $z$-symmetry axis in Fig. 1 . The plasma chamber is a copper cylinder of i.d. $=39$ and $\Delta z=90 \mathrm{~mm}$ inserted into the magnetic structure. It is closed by a plasma electrode with a 10 $\mathrm{mm}$ diameter extraction hole and by a coaxial waveguide or by a flat electrode with an off-axis microwave antenna. The walls of the plasma chamber are covered with $\mathrm{SiO}_{2}$ except for the waveguide entrance or for the antenna electrode. With the coaxial waveguide entrance a quasimicrowave resonator is created with a near perfect $E_{012}$ mode in the active plasma region while the off axis antenna yields a $H_{113}$ mode resonator, both at $6.7 \mathrm{GHz}$. The field amplitude distribution of both modes has been measured in test chambers without plasma and their resonance behavior has been reproduced with running source at slightly shifted frequencies by measuring the reflected microwave power. The results thus show that monomode microwave resonators can very successfully be employed in ECRIS for the production of highly charged ions. For the "NANOGAN', design ${ }^{3}$ such resonance behav-

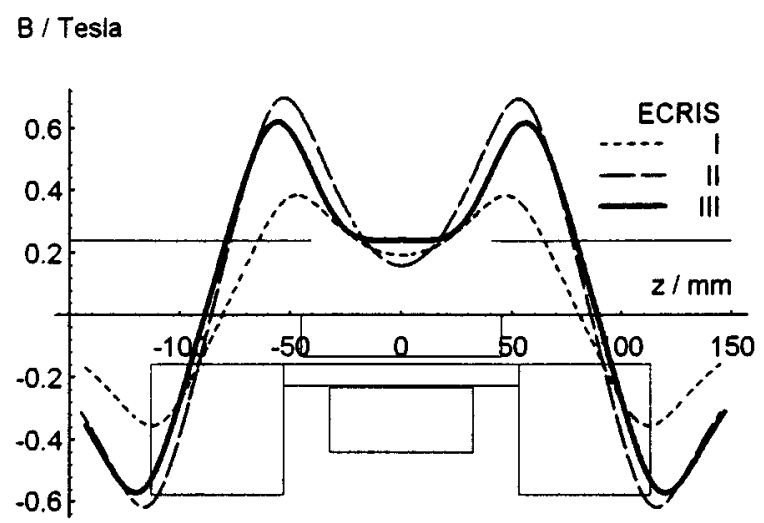

FIG. 1. Magnetic field components $B_{z}$ on the axis of symmetry of ECRIS I, II, and III with the indication of the positions of the radially magnetized rings for the mirror field, the hexapole, the additional central rings, and the plasma chamber. 
ior was suspected when measuring the ion output versus the microwave frequency, but no further proof was given.

The static magnetic fields are calculated by the method of magnetic polarization charges $\sigma=\mathbf{M} \cdot \mathbf{n}$ where $\mathbf{M}$ is the magnetization vector and $\mathbf{n}$ the surface normal unit vector for which the Lamy-Pauthenet integrals are a great simplification. ${ }^{4,5}$ Confined electrons in an ECRIS carry out millions of cyclotron revolutions around magnetic field lines. The Boris leap-frog algorithm ${ }^{6}$ takes advantage of the circular nature of these orbits. It is extended here to include the interaction with the oscillating electric microwave field of the two resonance modes, so that the ECR heating is completely incorporated. For cyclotron radii $<2 \mathrm{~mm}(10 \mathrm{keV}$ electrons at $\sim 0.24 \mathrm{~T}$ ) only 18 time steps are required for the correct numerical description of a cyclotron revolution. With $6 \mathrm{~h} \mathrm{CPU} \mathrm{time} \mathrm{of} \mathrm{a} \mathrm{small} \mathrm{workstation} \mathrm{it} \mathrm{is} \mathrm{thus} \mathrm{possible} \mathrm{to}$ pursue $10^{4}$ electrons over a time of $1 \mu \mathrm{s}$ in order to obtain satisfactory statistics concerning the density, the energy density, the energy spectrum, and the average energy of these electrons. To this end $10^{5}$ electrons are started with constant density in the whole volume of the plasma chamber with an energy of $10 \mathrm{eV}$ in arbitrary directions. After about $20 \mathrm{~ns}$ $\sim 80 \%$ of these electrons have hit a wall and are considered as lost because the secondary electron emission coefficient at the wall is set to zero here. Of the surviving electrons about $50 \%$ are on stable orbits with the initial $10 \mathrm{eV}$ without ever crossing an ECR zone and are therefore eliminated. The spatial distribution of the residual $\sim 10 \%$ of the initial electrons evolves from $20 \mathrm{~ns}$ to $1 \mu$ s due to the energy gain of the electrons and becomes quite stable near $1 \mu \mathrm{s}$. Different initial filling conditions yield the same results. It can therefore be assumed that rather realistic electron distributions are obtained after $1 \mu \mathrm{s}$ already for the present purposes from which the above quantities of interest are derived. In contrast to the above assumption, the secondary electron emission on our $\mathrm{SiO}_{2}$ walls may not be zero. We have therefore run some simulations with finite low energy electron emission upon electron impact at walls in the interval from $20 \mathrm{~ns}$ to $1 \mu \mathrm{s}$. The resulting low energy electrons stay in the outer lobes of the hexapolar field, are thus never accelerated, and do not alter the density- or energy-density distributions of electrons with higher energy, as obtained before with zero secondary electron emission.

By recording all simulated electron impacts on the inner walls of the plasma chamber from $t=100 \mathrm{~ns}$ to $T=1 \mu \mathrm{s}$ an impact pattern is obtained which exactly corresponds to the well known impact patterns as observed in all hexapolar ECRIS. Since ions are supposed to be present and to interact with surfaces (creating sputter traces) where the electrons are, this observation is taken as a first proof for the appropriate description of the plasma by the present simulation.

The energy spectrum of the electrons at $T=1 \mu \mathrm{s}$ is strongly dependent on the magnetic confinement, on the microwave mode and its electric field amplitude, and on the size of the ECR volume, i.e., the volume in which the ECR condition is fulfilled to within $\pm 1 \%$. For equal microwave power inside the plasma chamber the simulated energy spectra for the three ECRIS are shown in Fig. 2. They are obtained for electrons found in a cylinder of $34 \mathrm{~mm}$ length and

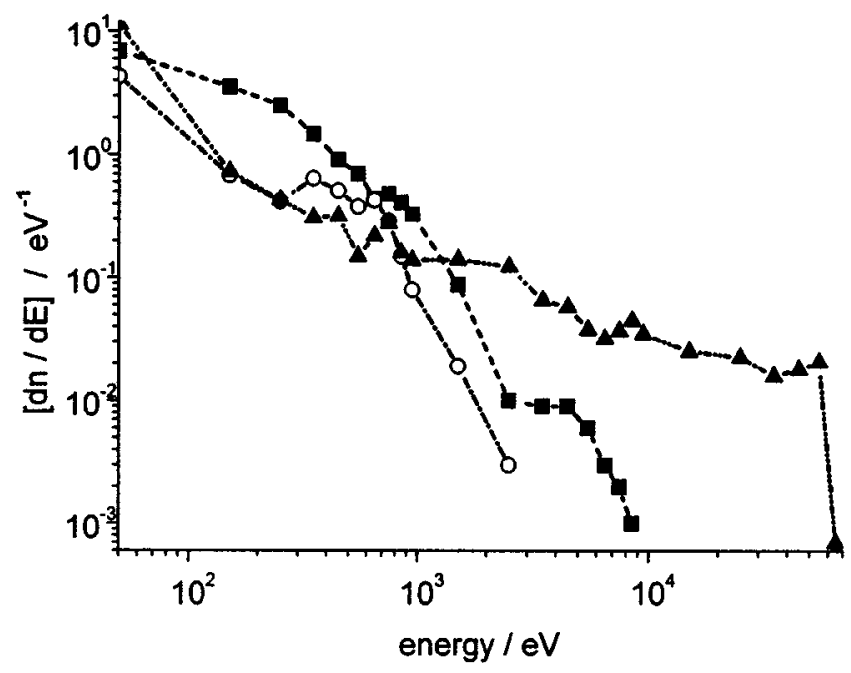

FIG. 2. Calculated electron energy spectra after $1 \mu$ s of microwave interaction time at the same electric field strength in the three ECRIS: $\mathbf{D C R I S ~ I ,}$ $\bigcirc$ ECRIS II, $\boldsymbol{\Delta}$ ECRIS III.

$20 \mathrm{~mm}$ diameter in contact with the extraction hole. The good but not extraordinary performance of ECRIS I is shown by the spectrum of charge states in Fig. 3 as obtained by measuring the ion currents after a separation magnet with symmetric foci at $570 \mathrm{~mm}$ where the Faraday cup of $15 \mathrm{~mm}$ diameter and the plasma electrode are located, respectively. A Faraday cup of $4 \mathrm{~mm}$ diameter reduces the currents by only $40 \%$ for all three sources. ECRIS I was later simulated to yield the electron energy spectrum in Fig. 2 which corresponds to its performance. In order to improve the situation, ECRIS II was built with higher $B_{z \text { max }}$ and smaller $B_{z \text { min }}$ for a high mirror ratio $B_{z \max } / B_{z \text { min }}$. Its performance was very disappointing, actually not even approaching ECRIS I, although 5 times more microwave power was injected. This

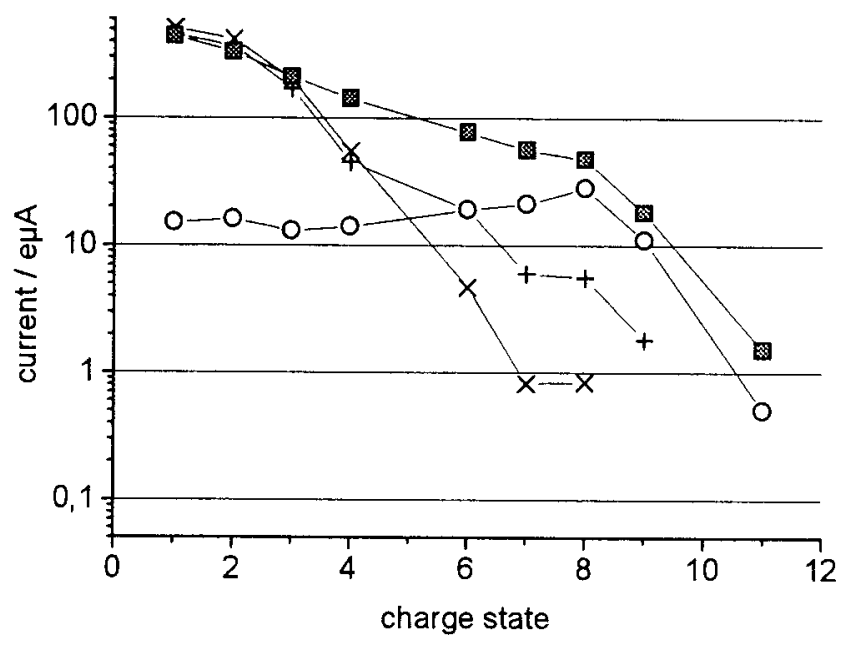

FIG. 3. Observed charge state distribution of extracted argon ion currents at $20 \mathrm{kV}$ extraction voltage, optimized for every charge state. + ECRIS I at

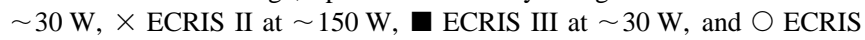
III at $\sim 12 \mathrm{~W}$ optimized for $\mathrm{Ar}^{9+}$. 
result did not improve when moving the center of the magnetic field of Fig. 1 by $10 \mathrm{~mm}$ towards the extraction with respect to the fixed plasma chamber. The subsequent simulation revealed indeed an electron energy spectrum with lower average energy in Fig. 2 due to a bad microwave coupling to the plasma caused by the steep field gradient at ECR resulting in a very small ECR volume. This is in agreement with earlier investigations on the influence of the magnetic field on the ECR heating efficiency. ${ }^{7}$ The simulation was therefore used to develop the final field configuration of ECRIS III in Fig. 1 with the goal of a great ECR volume and a predicted electron energy spectrum with high average energy in Fig. 2. ECRIS II was mechanically and magnetically converted to ECRIS III to yield the performance shown as charge state spectrum in Fig. 3. Actually this result was obtained after shifting the magnetic structure by $10 \mathrm{~mm}$ towards the extraction with respect to the fixed plasma chamber. The zone of high and hot electron density from the simulation, corresponding to high HCI density, is thus shifted towards the extraction hole so increasing the HCI currents considerably. Evidently the promising predictions of the simulation are fully confirmed by these results thus demonstrating the validity and usefulness of the model assumptions and of the simulation. The performance of ECRIS III is slightly better than that of the NANOGAN at $10 \mathrm{GHz},{ }^{3}$ and new simulations show that it can further be improved.

In order to further demonstrate the significance of the ECR volume, the outer radially magnetized rings of ECRIS III have been symmetrically moved in steps of $0.5 \mathrm{~mm}$ away from the center to systematically lower $B_{z \text { min }}$ with its large plateau. Starting with $B_{z \text { min }}>B_{\mathrm{ECR}}$ one so obtains the response of the performance of ECRIS III as measured by the $\mathrm{Ar}^{6+}$ output current in Fig. 4. This experiment was perfectly well predicted by the simulation when taking the average energy of the electrons, i.e., the ECR-heating efficiency as criterion, also plotted in Fig. 4. The extreme sensitivity on $B_{z \min }=B_{\mathrm{ECR}}$ is clearly demonstrated. This result also is an impressive verification of some of the ideas worked out in the excellent analysis of ECRIS by Alton and Smithe. ${ }^{8}$ They predicted a very good ECRIS performance for a flat $B_{z \text { min }}$-zone as successfully verified here for the first time. They used well known two-dimensional plasma codes while this conclusion is obtained here by a fully three-dimensional pursuit of the electrons, an approach which yields more in-

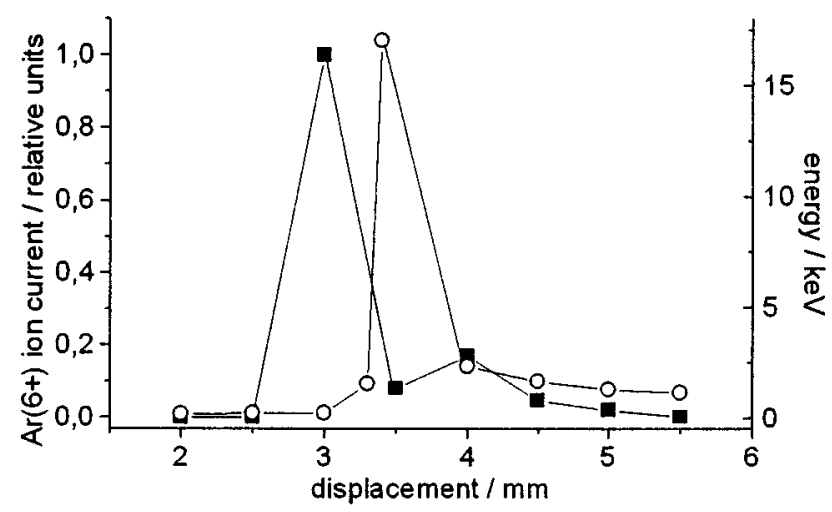

FIG. 4. $\mathrm{Ar}^{6+}$ ion currents of ECRIS III extracted at $20 \mathrm{kV}(\boldsymbol{\square})$, compared to the calculated average energy of the electrons after $1 \mu \mathrm{s}$ of microwave interaction time $(O)$, both as a function of the axial displacement of the outer radially magnetized rings. Note: $1 \mathrm{~mm}$ of displacement corresponds to $\Delta B_{z \min } \approx 8 \mathrm{mT}$.

formation on the electrons and should be valid for any collisionless plasma. By adding finite electron collision probabilities and the ionization, recombination, and motion of the ions in the now known spatial distributions of the electrons one can expect a rather complete steady state description of an ECRIS with further consequences for its better design.

This work is supported by the German Bundesministerium für Bildung, Wissenschaft, Forschung und Technologie under Contract No. 13N6776 4.

${ }^{1}$ R. Geller, Electron Cyclotron Resonance Ion Sources and ECR Plasmas (IOP, Bristol, 1966), p. 491 and references therein.

${ }^{2}$ K. Halbach, Nucl. Instrum. Methods Phys. Res. A 169, 1 (1980).

${ }^{3}$ P. Sortais, C. Bieth, P. Foury, N. Lecesne, R. Leroy, J. Mandin, C. Marry, J. Y. Pacquet, E. Robert, and A. C. C. Villari, in Proceedings of 2nd International Workshop on Electron Cyclotron Resonance Ion Sources, Saitama, Japan, April 1995, edited by M. Sekigushi and T. Nakagawa (INS, Tokio 182, 1995), p. 44-52.

${ }^{4}$ R. Pauthenet, J. Phys. (France) C1, 45 (1984).

${ }^{5}$ R. Pauthenet, J. Debernardi, M. Hakim, B. Jacquot, and T. Lamy, in Proceedings of the Fourth International Workshop on Electron Cyclotron Resonance Ion Sources, Grenoble, 1982 (IOP, Bristol, 1982), p. 131.

${ }^{6}$ Plasma Physics via Computer Simulation, edited by C. K. Birdsall and A. B. Langdon (IOP, Bristol, 1995).

${ }^{7}$ Y. Jongen, Proceedings of the Sixth International Electron Cyclotron Resonance Ion Source Workshop, Berkeley, CA, 1985, edited by C. Lyneis (unpublished), p. 238, PUB 5143.

${ }^{8}$ G. D. Alton and D. N. Smithe, Rev. Sci. Instrum. 65, 775 (1994). 